\title{
Manifolds of balance in planar ecological systems
}

\author{
Atheeta Ching ${ }^{a 1,2}$, Stephen Baigent ${ }^{a 1}$ \\ ${ }^{a}$ Department of Mathematics UCL, Gower Street, London, WC1E 6BT, UK. \\ DOI: https: //doi.org/10.1016/j. amc. 2019.04.047 \\ (C) 2019, Elsevier. Licensed under the Creative Commons \\ Attribution-NonCommercial-NoDerivatives 4.0 International \\ http: //creativecommons.org/licenses/by-nc-nd/4.0/
}

\begin{abstract}
In the classic 2-species Lotka-Volterra competition model, and more general competitive planar Kolmogorov models, there is a continuous curve called the carrying simplex that links all non-zero steady states and attracts all non-zero population densities. This curve is where the opposing processes of population growth and decline balance. In this paper, we use stability analysis and index theory to show that such a curve also exists when the interactions between two species are more general, such as co-operative or predator-prey, provided that reasonable biologically motivated conditions hold. For example, both species experience intraspecific competition and all population densities remain bounded for all time. We consider systems where there is at most one co-existence steady state. The 'balance manifold' is formed of heteroclinic orbits and attracts all non-zero population densities, but unlike its competitive analogue, the curve is no longer necessarily continuously differentiable.
\end{abstract}

Keywords: Kolmogorov system, carrying simplex, balance manifold, heteroclinic orbit

\section{Introduction}

Many biological and physical systems have opposing processes that lead eventually to a state where the processes are in a state of balance. For physical

\footnotetext{
Email address: Atheeta.Ching.11@ucl.ac.uk (Atheeta Ching ${ }^{a}$ )

${ }^{1}$ Declarations of interest: none

${ }^{2}$ Corresponding author
} 
systems, a classic example is a balance of forces which may result in an equilibrium, for example, a pendulum at rest at its lowest point.

In ecology, population density changes are due to a multitude of processes that contribute to population growth and decline. These processes are in turn controlled by factors such as fecundity, competition, co-operation, predation, environmental factors and so on. For many ecological systems, a long term state of balance will be achieved where these processes are in static or dynamic balance, although the underlying geometry of this state of balance may be extremely complex (for example, a strange attractor [1, 2, 3]). The purpose of this paper is to investigate a class of two-species deterministic ecological models and determine when a manifold of balance exists - balancing solutions coming out of the origin, and down from infinity in the phase

plane. Such models have a simple behaviour in that all orbits converge to a steady state on the balance manifold.

\section{Background}

For deterministic continuous-time single-species population models, the conditions for the existence of a unique positive environmental carrying capacity $K$ at which the population eventually settles are well-understood: the percapita growth rate is a continuous function $f: \mathbb{R}_{\geq 0} \rightarrow \mathbb{R}$ with $f(0)>0$, $f(x)<0$ for $x>K$ and $f^{-1}(0)=\{K\}$. Here we will use $\mathbb{R}_{\geq 0}=[0, \infty)$ and $\mathbb{R}_{>0}=(0, \infty)$. The key features behind a unique, attracting carrying capacity are: (i) the origin is repelling, (ii) infinity is repelling, and (iii) the positive equilibrium $K$ is unique. At the carrying capacity, there is a balance between the growth and decline of the population, meaning all non-zero population densities are attracted to this state.

An analogous concept of balance exists in higher dimensions. For competitive systems, Hirsch [4, 5] introduced carrying simplices which are hypersurfaces that asymptotically attract all non-zero initial population densities and contains all non-zero steady states. The most interesting property of a carrying simplex is that all non-trivial dynamics, such as periodic orbits, occur on it. The theory of carrying simplices for both continuous and discrete-time is an active research field, but to the best of our knowledge, all known results for carrying simplices relate to competitive [6, 4, 5, 7, 8] or type-K competitive systems [9]. For competitive Lotka-Volterra systems, the convexity of the carrying simplex has also been studied as it can imply properties of the system as a whole [10, 11, 12]. 
In [13] we have developed an analytic formula for an analogue of the carrying simplex which can also be applied to a class of non-competitve 2-species Lotka-Volterra systems. In this context it is referred to as a balance simplex, which can still be projected 1-to-1 and onto the unit simplex by radial projection. However many other properties no longer hold from carrying simplices. For example, the balance simplex is no longer $C^{1}$-continuous, nor is it the graph of a decreasing function (which all hold for the planar carrying simplex [10, 5, 14, 15]). The balance manifold that we introduce here is similar to the carrying simplex and the balance simplex, except we now do not require that the balance manifold projects radially 1-to-1 and onto the unit probability simplex.

\section{General Kolmogorov population models}

We examine a general planar Kolmogorov-type system

$$
\begin{aligned}
& \frac{d x_{1}}{d t}=F_{1}\left(x_{1}, x_{2}\right)=x_{1} f\left(x_{1}, x_{2}\right), \\
& \frac{d x_{2}}{d t}=F_{2}\left(x_{1}, x_{2}\right)=x_{2} g\left(x_{1}, x_{2}\right),
\end{aligned}
$$

where we only consider (1) on the phase space $\mathbb{R}_{\geq 0}^{2}$ and the functions $f, g$ : $\mathbb{R}_{\geq 0}^{2} \rightarrow \mathbb{R}_{\geq 0}^{2}$ are $C^{1}$-continuous on an open set containing $\mathbb{R}_{\geq 0}^{2}$. Such a system is often used to model the ecological dynamics of a closed habitat in which two species interact.

Our standing assumptions for (1) are:

A1 The origin $O$ and infinity are repellers;

A2 There are unique axial steady states $q_{1}=\left(\bar{x}_{1}, 0\right)$ and $q_{2}=\left(0, \bar{x}_{2}\right)$, $\bar{x}_{1}>0, \bar{x}_{2}>0$

A3 All steady states of (1) are hyperbolic, i.e. the eigenvalues of the Jacobian (see equation (5)) at all steady states have non-zero real parts;

A4 (Intraspecific competition)

$$
\frac{\partial f}{\partial x_{1}}(x)<0, \frac{\partial g}{\partial x_{2}}(x)<0, x=\left(x_{1}, x_{2}\right) \in \mathbb{R}_{\geq 0}^{2} .
$$


We denote the flow of the system by $\varphi(\cdot, t): \mathbb{R}_{\geq 0}^{2} \rightarrow \mathbb{R}_{\geq 0}^{2}$ or $\varphi_{t}(x)$ when $x$ is fixed. One important property of this system is that the axes are invariant (forwards and backwards in time). The interior $\mathbb{R}_{>0}^{2}$ also remains invariant.

Definition 3.1. We denote by $O^{+}(x)=\left\{\varphi_{t}(x): t \geq 0\right\}$ the forward orbit through $x$ and $O^{-}(x)=\left\{\varphi_{t}(x): t \leq 0\right\}$ the backward orbit through $x$. The orbit through $x$ is denoted by $O(x)=O^{+}(x) \cup O^{-}(x)$.

Definition 3.2. We denote by $\omega(x)$ the set of limit points of the forward orbit $O^{+}(x)$ and $\alpha(x)$ is the set of all limit points of the backward orbit $O^{-}(x)$.

Definition 3.3. A heteroclinic orbit is an orbit that joins two distinct steady states, and a homoclinic orbit is an orbit between the same steady state.

Definition 3.4. Let $p$ be a steady state of (1), we call any orbit which has its $\alpha$-limit equal to $p$ an unstable orbit of $p$. Similarly, any orbit which has its $\omega$-limit equal to $p$ is called a stable orbit of $p$.

Definition 3.5. Let $N$ a neighbourhood of $p \in U$. Then the local stable manifold of $p$ relative to $N$, written $W_{l o c}^{s}(p)=\left\{x \in N: O^{+}(x) \subset\right.$ $N$ and $\varphi_{t}(x) \rightarrow p$ as $\left.t \rightarrow \infty\right\}$. The global stable manifold is $W^{s}(p)=$ $\bigcup_{t<0} \varphi_{t}\left(W_{l o c}^{s}(p)\right)$. The local unstable manifold of $p$ relative to $N$, written $W_{l o c}^{\bar{u}}(p)=\left\{x \in N: O^{-}(x) \subset N\right.$ and $\varphi_{t}(x) \rightarrow p$ as $\left.t \rightarrow-\infty\right\}$. The global unstable manifold is $W^{u}(p)=\bigcup_{t \geq 0} \varphi_{t}\left(W_{l o c}^{u}(p)\right)$.

Definition 3.6. The basin of attraction of $p$ is $\mathcal{B}(p)=W^{s}(p)$. Similarly, the basin of repulsion of $p$ is the open set $\mathcal{R}(p)=W^{u}(p)$.

To elucidate what its means to say that infinity is a repeller for (1) we make a co-ordinate change to bring infinity into view. We define the inversion map $X=\left(X_{1}, X_{2}\right): \mathbb{R}_{\geq 0}^{2} \backslash O \rightarrow \mathbb{R}_{\geq 0}^{2}$ via

$$
X_{1}(x)=\frac{x_{1}}{x_{1}^{2}+x_{2}^{2}}, X_{2}(x)=\frac{x_{2}}{x_{1}^{2}+x_{2}^{2}} .
$$

Then $X$ maps infinity in $x_{1}, x_{2}$ co-ordinates to the origin in $X_{1}, X_{2}$ coordinates. Infinity is repelling in (1) when the origin of the transformed system

$$
\begin{aligned}
& \dot{X}_{1}=\left(X_{2}^{2}-X_{1}^{2}\right) \bar{F}_{1}\left(X_{1}, X_{2}\right)-2 X_{1} X_{2} \bar{F}_{2}\left(X_{1}, X_{2}\right) \\
& \dot{X}_{2}=\left(X_{1}^{2}-X_{2}^{2}\right) \bar{F}_{2}\left(X_{1}, X_{2}\right)-2 X_{1} X_{2} \bar{F}_{1}\left(X_{1}, X_{2}\right),
\end{aligned}
$$


is an unstable node, where $\bar{F}_{i}\left(X_{1}, X_{2}\right)=F_{i}\left(x_{1}, x_{2}\right)$ for $i=1,2$. In this case there is an $\epsilon>0$ and arc $\left\{\left(X_{1}, X_{2}\right) \in \mathbb{R}_{\geq 0}^{2}: X_{1}^{2}+X_{2}^{2}=\epsilon\right\}$ that all transformed orbits cross outwards. Back in the original co-ordinates, this means that there is an arc $\left\{\left(x_{1}, x_{2}\right) \in \mathbb{R}_{\geq 0}^{2}: x_{1}^{2}+x_{2}^{2}=\epsilon^{-1}\right\}$ that all orbits from infinity cross inwards.

To obtain the basin of repulsion of infinity in (1) we can alternatively find the basin of attraction of the origin in (4) and map back to $x_{1}, x_{2}$ co-ordinates.

Definition 3.7. We define a balance manifold for (1) to be a globally attracting (on $\mathbb{R}_{\geq 0}^{2} \backslash\{0\}$ ), compact, connected curve that is equal to the union of the boundaries of the basins of repulsion of the origin and of infinity.

Remark 1. Note that since the balance manifold is globally attracting on $\mathbb{R}_{\geq 0}^{2} \backslash\{0\}$, it is invariant for the flow of $(1)$ and necessarily contains all nonzero steady states.

The balance manifold $\Sigma$ is analogous to the carrying simplex which exists in the competitive case where $\frac{\partial f}{\partial x_{2}}<0$ and $\frac{\partial g}{\partial x_{1}}<0$ in $\mathbb{R}_{\geq 0}^{2}$ [5]. Conceptually, we want the balance manifold to separate the basins of repulsion of the origin and of infinity as shown in Figure 1 .

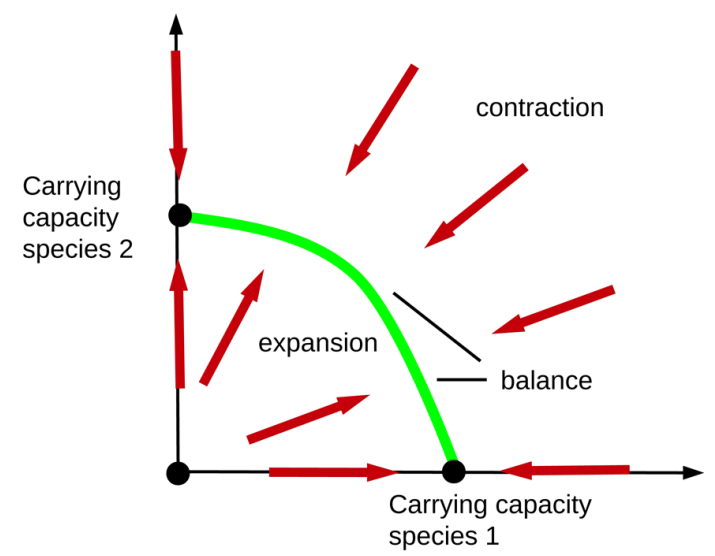

Figure 1: The balance manifold. [COLOUR: ONLINE ONLY]

The thick (red) arrows show the direction of the dynamics in different parts of the phase plane of a 2-species system. The solid (green) curve is the balance manifold.

A simple, but important, consequence of assumption A4 is: 
Lemma 3.1. Under the assumption (2) there can be no interior closed orbits for (1).

Proof. Via the Dulac Theorem [16] with Dulac function $B(x)=\left(x_{1} x_{2}\right)^{-1}$,

$$
\operatorname{div}\left(B\left(x_{1} f, x_{2} g\right)\right)=\frac{1}{x_{2}} \frac{\partial f}{\partial x_{1}}+\frac{1}{x_{1}} \frac{\partial g}{\partial x_{2}}<0, x=\left(x_{1}, x_{2}\right) \in \mathbb{R}_{>0}^{2},
$$

and so there can be no interior closed orbits.

While this rules out interior homoclinic orbits, it does not rule out homoclinic orbits from a boundary steady state. For this possibility we have

Lemma 3.2. Under the assumptions A1-A4 it is not possible for the unstable orbit of an axial saddle steady state of (1) to be a homoclinic orbit.

Proof. Without loss of generality assume the axial saddle steady state with a homoclinic orbit is $q_{1}$. By assumption A3 the saddle $q_{1}$ has a one-dimensional unstable manifold $W^{u}\left(q_{1}\right)$ and a one-dimensional stable manifold $W^{s}\left(q_{1}\right)$. Let $x^{0}=\left(x_{1}^{0}, x_{2}^{0}\right) \in W^{u}\left(q_{1}\right)$ with $x_{2}^{0}>0$ and $O^{+}\left(x^{0}\right)$ be the forward orbit through $x^{0}$. Then if the unstable orbit of $q_{1}$ is a homoclinic orbit, $\varphi_{t}\left(x^{0}\right) \rightarrow q_{1}$ as $t \rightarrow \infty$, so that $x^{0} \in W^{s}\left(q_{1}\right)=\left\{(x, 0): x \in \mathbb{R}_{>0}\right\}$, a contradiction to $x_{2}^{0}>0$.

It will be useful to note the Jacobian of the system (1):

$$
\mathcal{J}\left(x_{1}, x_{2}\right)=\left(\begin{array}{cc}
f\left(x_{1}, x_{2}\right)+x_{1} \frac{\partial f}{\partial x_{1}}\left(x_{1}, x_{2}\right) & x_{1} \frac{\partial f}{\partial x_{2}}\left(x_{1}, x_{2}\right) \\
x_{2} \frac{\partial g}{\partial x_{1}}\left(x_{1}, x_{2}\right) & g\left(x_{1}, x_{2}\right)+x_{2} \frac{\partial g}{\partial x_{2}}\left(x_{1}, x_{2}\right)
\end{array}\right) .
$$

At the origin $O$

$$
\mathcal{J}(0,0)=\left(\begin{array}{cc}
f(0,0) & 0 \\
0 & g(0,0)
\end{array}\right)
$$

so to satisfy assumption A1 we require $f(0,0)>0$ and $g(0,0)>0$ for the origin to be repelling.

Assumption A2 requires that (1) has a unique positive steady state on each axis, which will be the carrying capacity of each individual species. Consider the axial state $q_{2}=\left(0, \bar{x}_{2}\right)$ where $\bar{x}_{2}>0$ and $g\left(q_{2}\right)=0$. The Jacobian here is

$$
\mathcal{J}\left(q_{2}\right)=\left(\begin{array}{cc}
f\left(q_{2}\right) & 0 \\
\bar{x}_{2} \frac{\partial g}{\partial x_{1}}\left(q_{2}\right) & \bar{x}_{2} \frac{\partial g}{\partial x_{2}}\left(q_{2}\right)
\end{array}\right) .
$$


Assumption A1 ensures that $q_{2}$ is asymptotically stable on the $x_{1}$-axis. The invariant $x_{2}$-axis has the associated eigenvalue $\bar{x}_{2} \frac{\partial g}{\partial x_{2}}\left(q_{2}\right)$, and so we require $\frac{\partial g}{\partial x_{2}}\left(q_{2}\right)<0$. We also assume $f\left(q_{2}\right) \neq 0$ to avoid a non-hyperbolic steady state. The sign of $f\left(q_{2}\right)$ determines whether this axial steady state is a saddle point or a stable node.

Similarly, at the other axial steady state, $q_{1}=\left(\bar{x}_{1}, 0\right)$, we require $\frac{\partial f}{\partial x_{1}}\left(q_{1}\right)<0$, and the sign of $g\left(q_{1}\right)$ determines whether $q_{1}$ is a saddle point or a stable node.

By considering the Poincaré index of steady states (see, for example, [16]), we can determine the possible stability types of the steady state of (1).

Definition 3.8. Let $\Gamma$ be a smooth ${ }^{3}$, closed curve traversed anti-clockwise and consisting of only ordinary points of the vector field (i.e. no steady states). Let $\theta$ be the angle between the flow of the vector field at a point on $\Gamma$ and the positive horizontal axis. Consider a point $x^{0} \in \Gamma$. Since the flow is continuous, as we traverse around $\Gamma$, back to $x^{0}$, the variation of $\theta$ will be a multiple of $2 \pi$, say $2 k \pi ; k \in \mathbb{Z}$. The index of $\Gamma$, denoted $I_{\Gamma}$, is $k$.

We let $[\theta]_{\gamma}$ denote the change in the angle $\theta$ as we traverse a curve $\gamma$, so that $I_{\Gamma}=\frac{[\theta]_{\Gamma}}{2 \pi}$. Let $\Gamma$ be the closed curve orientated anticlockwise as shown in Figure 2, There are different components to $\Gamma=\Gamma_{0} \cup \Gamma_{1} \cup \Gamma_{a} \cup \Gamma_{2}$. In this case, since $\Gamma$ contains only ordinary points, its index is zero. As the origin is an unstable node, $[\theta]_{\Gamma_{0}}=\frac{\pi}{2}$.

To calculate the change in angle along the component $\Gamma_{a}$, we bring infinity into view via the inversion map (3). Then infinity is repelling in (1) when the origin of the system in $X_{1}, X_{2}$ co-ordinates is an unstable node. In that case there is an $\epsilon>0$ and arc $\left\{\left(X_{1}, X_{2}\right) \in \mathbb{R}_{>0}^{2}: X_{1}^{2}+X_{2}^{2}=\epsilon\right\}$ that all transformed orbits cross outwards. Back in the original co-ordinates, this means that there is an arc $\left\{\left(x_{1}, x_{2}\right) \in \mathbb{R}_{\geq 0}^{2}: x_{1}^{2}+x_{2}^{2}=\epsilon^{-1}\right\}$ that all orbits from infinity cross inwards. Hence $[\theta]_{\Gamma_{a}}=-\frac{\pi}{2}$.

Now suppose that $q_{1}$ is a saddle, then $[\theta]_{\Gamma_{1}}=-\pi$, whereas if $q_{1}$ is a stable node, $[\theta]_{\Gamma_{1}}=+\pi$. Suppose that both $q_{1}$ and $q_{2}$ are saddles. Then the total angle change should be zero, since there are no steady states inside $\Gamma$, but we find $[\theta]_{\Gamma}=\frac{\pi}{2}-\frac{\pi}{2}-\pi-\pi=-2 \pi$. Hence it is not possible that both of $q_{1}, q_{2}$ are saddles. Similarly if $q_{1}, q_{2}$ cannot both be stable nodes, since then we would have $[\theta]_{\Gamma}=2 \pi$. Thus the only possible case is when one axial steady

\footnotetext{
${ }^{3}$ See [17] for the extension to piecewise smooth curves.
} 


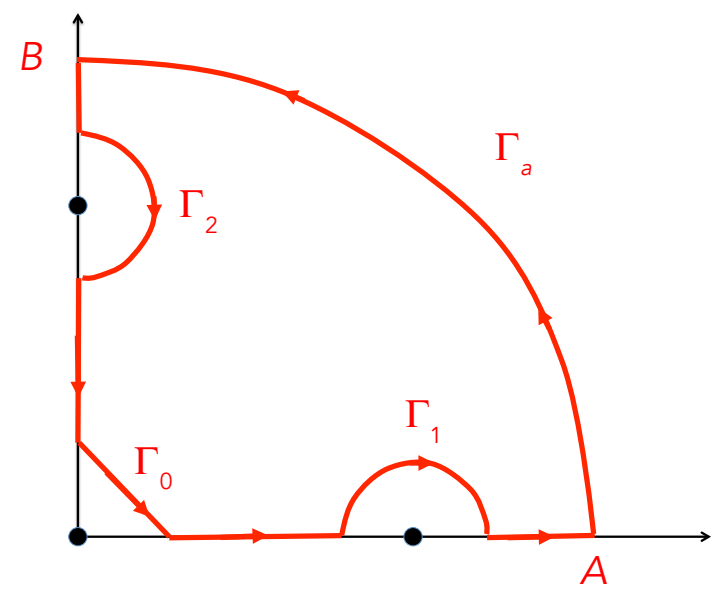

Figure 2: A closed curve enclosing no steady states. [COLOUR: ONLINE ONLY]

$\Gamma=\Gamma_{0} \cup \Gamma_{1} \cup \Gamma_{a} \cup \Gamma_{2}$ is the thick (red) closed curve traversed anticlockwise, looping around each axial steady state. $\Gamma_{0}$ is sufficiently close to the origin such that the vector field is pointing away from the origin, and $\Gamma_{a}$ is arbitrarily far from the origin such that $\Gamma$ contains all possible interior steady states.

state is a saddle and the other is a stable node.

On the other hand, if there are interior steady states, then with $\Gamma_{a}$ chosen so that all interior steady states lie inside $\Gamma$, we find that the sum of the indices of the interior steady states is the index of $\Gamma$. Hence when there is a unique interior steady state $x^{*}$, the index of $\Gamma$ is then equal to the index of $x^{*}$. Now there are more possibilities.

If both $q_{1}, q_{2}$ are saddles, then the index of $x^{*}$ is equal to plus one, so that $x^{*}$ cannot be a saddle. With the assumption of $x^{*}$ being hyperbolic and intraspecific competition (preventing interior closed orbits) it follows that $x^{*}$ must be stable and a node or spiral. If both $q_{1}, q_{2}$ are stable nodes, then the index of $x^{*}$ is equal to minus one, so that $x^{*}$ must be a saddle. Lastly, the case where one axial steady state is a saddle and the other is a stable node is not possible; $x^{*}$ would have an index of zero, contradicting its hyperbolicity.

To summarise

Lemma 3.3. For the system (1) under the assumptions A1-A4: 
1. If there is no interior steady state, one axial steady state must be a saddle and the other a stable node.

2. If there is a unique interior steady state, then

(a) If both axial fixed points are saddles, the interior fixed point is not a saddle, and so it must be a stable node or a stable spiral.

(b) If both axial fixed points are stable nodes, the interior fixed point is a saddle.

(c) It is not possible that one axial fixed point is a saddle if the other is a stable node.

\section{Case 1: no interior fixed point}

Without loss of generality, let us suppose that $q_{1}$ is a saddle and $q_{2}$ a stable node.

Lemma 4.1. Consider the system (1), under the assumptions A1-A4. Assume there is no interior steady state. Suppose one of these axial steady states is a saddle point, and the other is a stable node. Then the balance manifold is formed of the unique heteroclinic orbit connecting the axial steady states (along with these steady states).

Proof. Since we are only considering hyperbolic steady states, the saddle $q_{1}$ has a one-dimensional unstable manifold $W^{u}\left(q_{1}\right)$. Let $x^{0} \in W^{u}\left(q_{1}\right)$ in $\mathbb{R}_{>0}^{2}$ and $O^{+}\left(x^{0}\right)$ be the forward orbit through $x^{0}$. By assumption $\mathrm{A} 1, O^{+}\left(x^{0}\right)$ is bounded and so by the Poincaré-Bendixson theorem $\omega\left(x^{0}\right)$ contains a steady state, say $p$. By Lemmas 3.1 and 3.2 , there are no homoclinic orbits, so $p \neq q_{1}$. Moreover, $O$ is repelling so $p \neq O$. This leaves $p=q_{2}$. Then since $q_{2} \in \omega\left(x^{0}\right)$ there exists a $t_{k} \rightarrow \infty$ such that $\varphi_{t_{k}}\left(x^{0}\right) \rightarrow q_{2}$ and hence a $K^{\prime}$ such that $\varphi_{t_{K^{\prime}}}\left(x^{0}\right) \in \mathcal{B}\left(q_{2}\right)$ and $\varphi_{t}\left(x^{0}\right) \in \mathcal{B}\left(q_{2}\right)$ for all $t>t_{K^{\prime}}$, and so $\omega\left(x^{0}\right)=\left\{q_{2}\right\}$. Hence $\overline{O^{+}\left(x^{0}\right)}$ is a curve that connects $x^{0} \in W^{u}\left(q_{1}\right)$ to $q_{2}$ and we obtain a heteroclinic orbit $\mathcal{H}=W^{u}\left(q_{1}\right) \backslash\left\{q_{1}\right\}$.

Next we show that $\overline{\mathcal{H}}=\partial \mathcal{R}(0)=\partial \mathcal{R}(\infty) . \overline{\mathcal{H}}$ divides $\mathbb{R}_{>0}^{2}$ into two disjoint connected and invariant components, say $\mathcal{H}^{-}$containing $O$ and $\mathcal{H}^{+}=\mathbb{R}_{\geq 0}^{2} \backslash$ $\left(\overline{\mathcal{H}} \cup \mathcal{H}^{-}\right)$. Let $x^{0} \in \mathcal{H}^{-}$and consider $O^{-}\left(x^{0}\right)$. Since $x^{0} \notin \overline{\mathcal{H}}=W^{u}\left(q_{1}\right) \cup\left\{q_{2}\right\}$, there is no subsequence $t_{k} \rightarrow-\infty$ with $\varphi_{t_{k}}\left(x^{0}\right) \rightarrow q_{1}$ and hence $q_{1} \notin \alpha\left(x^{0}\right)$. 
By the Poincaré-Bendixson theorem we must have $O \in \alpha\left(x^{0}\right)$. Since $O$ is a stable node backwards in time, we see that $\alpha\left(x^{0}\right)=\{O\}$. Hence $\mathcal{H}^{-}=\mathcal{R}(O)$.

Next we map $\mathcal{H}^{+}$to $X\left(\mathcal{H}^{+}\right)$using the inversion $(3)$ and consider the transformed dynamics (4). Examining these dynamics near the transformed steady states shows that their stability types remain the same. This gives the same phase portrait topology as the previous paragraph, and we conclude that $\mathcal{H}^{+}=\mathcal{R}(\infty)$

Hence for the case where $q_{1}$ is a saddle and $q_{2}$ a stable node, we have the balance manifold

$$
\overline{\mathcal{H}}=\partial \mathcal{R}(0)=\partial \mathcal{R}(\infty)=W^{u}\left(q_{1}\right) \cup\left\{q_{2}\right\} .
$$

To summarise, we have shown:

Theorem 4.2. For a balance manifold to exist in the case where (1) has no interior steady state, the following conditions are sufficient:

1. $f(0,0)>0, g(0,0)>0$; the origin is repelling.

2. Infinity is repelling.

3. There exists a unique axial steady state $q_{2}=\left(0, \bar{x}_{2}\right)$ on the positive $x_{2}$-axis satisfying $g\left(q_{2}\right)=0, \frac{\partial g}{\partial x_{2}}\left(q_{2}\right)<0$ and $f\left(q_{2}\right) \neq 0$.

4. There exists a unique axial steady state $q_{1}=\left(\bar{x}_{1}, 0\right)$ on the positive $x_{1}$-axis satisfying $f\left(q_{1}\right)=0, \frac{\partial f}{\partial x_{1}}\left(q_{1}\right)<0$ and $g\left(q_{1}\right) \neq 0$.

5. $f\left(q_{2}\right) g\left(q_{1}\right)<0$; the axial steady states are of different stability types and are hyperbolic.

\section{Case 2: a unique interior fixed point}

We now consider the case where there is a unique interior steady state $x^{*}=$ $\left(x_{1}^{*}, x_{2}^{*}\right)$. The first four conditions from Theorem 4.2 are still required. The Jacobian at the interior steady state is:

$$
\mathcal{J}\left(x_{1}^{*}, x_{2}^{*}\right)=\left(\begin{array}{ll}
x_{1}^{*} \frac{\partial f}{\partial x_{1}}\left(x_{1}^{*}, x_{2}^{*}\right) & x_{1}^{*} \frac{\partial f}{\partial x_{2}}\left(x_{1}^{*}, x_{2}^{*}\right) \\
x_{2}^{*} \frac{\partial g}{\partial x_{1}}\left(x_{1}^{*}, x_{2}^{*}\right) & x_{2}^{*} \frac{\partial g}{\partial x_{2}}\left(x_{1}^{*}, x_{2}^{*}\right)
\end{array}\right) .
$$


By assumption $x^{*}$ is hyperbolic and it may be a node or a saddle. However, when $x^{*}$ is a node, it is necessarily asymptotically stable due to assumption A4, through the condition (2).

Lemma 5.1. Let $x^{*}$ be the unique interior fixed point of the system (1) under the assumptions A1-A4. Suppose that both axial steady states are saddle points. Then the balance manifold is formed of the two heteroclinic orbits connecting the axial steady states to $x^{*}$ (along with these steady states).

Proof. Each axial steady state is a hyperbolic saddle with one-dimensional stable and unstable manifolds. Let $W^{u}\left(q_{1}\right)$ be the unstable manifold of $q_{1}$ and choose $x^{0} \in W^{u}\left(q_{1}\right)$ in $\mathbb{R}_{>0}^{2}$. By the Poincaré-Bendixson theorem, $\omega\left(x^{0}\right)$ must contain a steady state $p$, and $p \neq O$ since $O$ is repelling and $p \neq q_{1}$ by Lemma 3.2. Moreover $p \neq q_{2}$. To see this, note that for $q_{2} \in \omega\left(x^{0}\right)$ there exists $t_{k} \rightarrow \infty$ as $k \rightarrow \infty$ with $\varphi_{t_{k}}\left(x^{0}\right) \rightarrow q_{2}$. Suppose that $\varphi_{t_{k}}\left(x^{0}\right) \notin W^{s}\left(q_{2}\right)$ for any $k$. Then $\varphi_{t}\left(x^{0}\right) \notin W^{s}\left(q_{2}\right)$ for any $t$ since $W^{s}\left(q_{2}\right)$ is exactly the invariant $x_{2}$-axis. Noting the Hartman-Grobman [16] theorem, it follows that $\varphi_{t_{k}}\left(x^{0}\right) \not \rightarrow q_{2}$, a contradiction. Hence we are left with $p=x^{*}$, which is asymptotically stable. Since $O^{+}\left(x^{0}\right) \cap \mathcal{B}\left(x^{*}\right) \neq \emptyset$, there is a heteroclinic connection $\mathcal{H}_{2}=W^{u}\left(q_{1}\right) \backslash\left\{x^{*}\right\}$ between $q_{1}$ and $x^{*}$. Similarly there is a heteroclinic connection $\mathcal{H}_{3}=W^{u}\left(q_{2}\right) \backslash\left\{x^{*}\right\}$ linking $q_{2}$ and $x^{*}$.

Next we show that $\partial \mathcal{R}(0)=\overline{W^{u}\left(q_{1}\right) \cup W^{u}\left(q_{2}\right)}$. Let $\mathcal{H}=\mathcal{H}_{2} \cup \mathcal{H}_{3} . \overline{\mathcal{H}}$ divides $\mathbb{R}_{\geq 0}^{2}$ into two disjoint connected and invariant components, say $\mathcal{H}^{-}$containing $O$ and $\mathcal{H}^{+}=\mathbb{R}_{>0}^{2} \backslash\left(\overline{\mathcal{H}} \cup \mathcal{H}^{-}\right)$. Let $x^{0} \in \mathcal{H}^{-}$. We will show that $\alpha\left(x^{0}\right)=\{O\}$. By the Poincaré-Bendixson theorem, $\alpha\left(x^{0}\right)$ contains a steady state $p$ and as $x^{*}$ is attracting $p \neq x^{*}$. Moreover, $p \notin\left\{q_{1}, q_{2}\right\}$ since $x^{0} \notin W^{u}\left(q_{1}\right) \cup W^{u}\left(q_{2}\right)$. Hence $p=O$ and since $O$ is an unstable node, $\alpha(x)=\{O\}$ and $\mathcal{H}^{-}=\mathcal{B}(O)$, $\overline{\mathcal{H}}=\partial \mathcal{R}(O)$. As in Lemma 4.1, we may use the inversion map (3) to establish that $\mathcal{H}^{+}=\mathcal{R}(\infty)$ and $\overline{\mathcal{H}}=\partial \mathcal{R}(\infty)$.

We conclude that in this case the balance manifold

$$
\overline{\mathcal{H}}=\partial \mathcal{R}(0)=\partial \mathcal{R}(\infty)=\overline{W^{u}\left(q_{1}\right) \cup W^{u}\left(q_{2}\right)} .
$$

Lemma 5.2. Let $x^{*}$ be the unique interior fixed point of the system (1), under assumptions A1-A4. Suppose both axial steady states are stable nodes. 
Then $x^{*}$ is a saddle point and the two unstable orbits of $x^{*}$ have different $\omega$-limits, each equal to exactly one of the axial steady states.

Proof. That $x^{*}$ is a saddle follows from the Index Theorem. By the PoincaréBendixson theorem each unstable orbit of $x^{*}$ has a $\omega$-limit set that contains a steady state, which in neither case can be $O$, since $O$ is an unstable node, nor $x^{*}$, since there are no interior homoclinic orbits by Lemma 3.1. Consider one of the unstable orbits of $x^{*}$, call it $\gamma_{1}$. Then since $\gamma_{1}$ has $\omega$-limit in $\left\{q_{1}, q_{2}\right\}$, $\gamma_{1} \cap\left(\mathcal{B}\left(q_{1}\right) \cup \mathcal{B}\left(q_{2}\right)\right) \neq \emptyset$. Hence $\gamma_{1}$ is a heteroclinic orbit connecting $x^{*}$ to either $q_{1}$ or $q_{2}$. Similarly for the other unstable orbit $\gamma_{2}$. Suppose that $\gamma_{1}$ and $\gamma_{2}$ connect to the same $\omega$-limit, say $q_{1}$. These two heteroclinic orbits $\gamma_{1}, \gamma_{2}$ enclose a bounded and invariant region $R^{*}$ and $x^{*} \in \overline{R^{*}}$. Since $x^{*}$ is hyperbolic, $W^{s}\left(x^{*}\right) \cap R^{*} \neq \emptyset$. Choose $x^{0} \in W^{s}\left(x^{*}\right) \cap R^{*}$. By the Poincaré-Bendixson theorem, $\alpha\left(x^{0}\right)$ contains a steady state, say $p . p \neq x^{*}$ since we can have no interior closed orbits, and $p \neq q_{2}$ because $\overline{R^{*}}$ is invariant and disjoint from the $x_{2}$-axis. Hence we must have $p=q_{1}$. But this is not possible because $q_{1}$ is a stable node.

Next we show that $\partial \mathcal{R}(0)=\overline{W^{u}\left(x^{*}\right)}$. Let $\mathcal{H}=\gamma_{1} \cup \gamma_{2} . \overline{\mathcal{H}}$ divides $\mathbb{R}_{\geq 0}^{2}$ into two disjoint connected and invariant components, say $\mathcal{H}^{-}$containing $O$ and $\mathcal{H}^{+}=\mathbb{R}_{\geq 0}^{2} \backslash\left(\overline{\mathcal{H}} \cup \mathcal{H}^{-}\right)$. Let $x^{0} \in \mathcal{H}^{-}$. We will show that $\alpha\left(x^{0}\right)=\{O\}$. By the Poincaré-Bendixson theorem, $\alpha\left(x^{0}\right)$ contains a steady state $p$ and as $q_{1}, q_{2}$ are stable nodes $p \notin\left\{q_{1}, q_{2}\right\}$. Moreover, $p \neq x^{*}$ since $x^{0} \notin W^{u}\left(x^{*}\right)$. Hence $p=O$. The remainder of the proof is similar to that of Lemma 5.1.

In this case the balance manifold

$$
\overline{\mathcal{H}}=\partial \mathcal{R}(0)=\partial \mathcal{R}(\infty)=\overline{W^{u}\left(x^{*}\right)} .
$$

To summarise, we have shown:

Theorem 5.3. For a balance manifold (connecting all non-zero steady states) to exist for the system (1) in the case where there is a unique interior steady state $x^{*}$, the following conditions are sufficient:

1. $f(0,0)>0, g(0,0)>0$; the origin is repelling.

2. Infinity is repelling. 
3. There exists a unique axial steady state $q_{2}$ on the $x_{2}$-axis satisfying $g\left(q_{2}\right)=0, \frac{\partial g}{\partial x_{2}}\left(q_{2}\right)<0$ and $f\left(q_{2}\right) \neq 0$.

4. There exists a unique axial steady state $q_{1}$ on the $x_{1}$-axis satisfying $f\left(q_{1}\right)=0, \frac{\partial f}{\partial x_{1}}\left(q_{1}\right)<0$ and $g\left(q_{1}\right) \neq 0$.

5. $f\left(q_{2}\right) g\left(q_{1}\right)>0$; both axial steady states are of the same stability-type and are hyperbolic.

6. There exists a unique interior steady state $x^{*}>0$ satisfying $f\left(x^{*}\right)=$ $0=g\left(x^{*}\right)$.

7. There is intraspecific competition for each species; $\frac{\partial f}{\partial x_{1}}<0, \frac{\partial g}{\partial x_{2}}<0$ in $\mathbb{R}_{>0}^{2}$.

\section{Structural stability}

For dynamical systems, it is important to ask whether they are structurally stable [18], especially those which are used to model real systems [19]. A system is structurally stable if it remains topologically unchanged when the system (i.e. its vector field) is affected by a small perturbation. Consider two systems for $x \in \mathbb{R}^{2}$ in some closed region $\Omega \subset \mathbb{R}^{2}$

$$
\begin{aligned}
& \frac{d x}{d t}=F(x), \\
& \frac{d x}{d t}=G(x) .
\end{aligned}
$$

The 'distance' between these two systems can be measured by the following metric [20, 21]:

Definition 6.1. The distance between systems $(9)$ and $(10)$ in a closed region $\Omega \subset \mathbb{R}^{2}$ is given by:

$$
d_{1}=\sup _{x \in \Omega}\{\|F(x)-G(x)\|+\|\mathcal{J}(F)-\mathcal{J}(G)\|\}
$$

where $\mathcal{J}$ is the Jacobian matrix and the norm is the Frobenius norm in the relevant dimension. The systems are $\varepsilon$-close in $\Omega$ if $d_{1} \leq \varepsilon$; in which case (10) is considered a small perturbation of (9).

Theorem 6.1 (Andronov and Pontryagin [21, 20]). A smooth dynamical system (9) is structurally stable in a region $\Omega \subset \mathbb{R}^{2}$ if and only if: 
1. it has a finite number of equilibria and limit cycles in $\Omega$, all of which are hyperbolic,

2. there are no saddle points with a homoclinic orbit and there are no heteroclinic orbits connecting two saddle points in $\Omega$.

The system (1) we consider with conditions from Theorems 4.2 or 5.3 is therefore structurally stable as it satisfies the conditions of Theorem 6.1. Indeed, we restrict all steady states to be hyperbolic. In the case where two steady states are saddle points, we know there must be three non-zero steady states total. In Theorem 5.3 we imposed conditions to ensure both axial steady states will be the saddle points in this scenario. In Lemma 5.1. we showed there is no heteroclinic orbit between these saddle points.

Therefore the balance simplex we have found (composed of heteroclinic orbits) is also structurally stable in the sense that it still exists when the system is affected by a small perturbation. This means the balance simplex is not a rare or atypical structure, but occurs in a range of systems, characterised by the conditions in Theorems 4.2 and 5.3 .

\section{Example models}

\subsection{Lotka-Volterra equations}

In [13], we considered a general Lotka-Volterra 2-species model where the intrinsic growth rates and intraspecfic interaction coeffecients are all equal

to 1 for both species. In this case, we can find an analytic solution for the balance manifold. This system is

$$
\begin{aligned}
& \frac{d x_{1}}{d t}=x_{1}\left(1-x_{1}-\alpha x_{2}\right), \\
& \frac{d x_{2}}{d t}=x_{2}\left(1-\beta x_{1}-x_{2}\right),
\end{aligned}
$$

where the interspecific interactions are $\alpha$ and $\beta$ (which can be any sign). The solution is given in terms of Gaussian hypergeometric functions and a variable $T \in[0, \infty)$, where $T=\tan (\theta)$ from polar co-ordinates. Consider the case where $\beta<1$, and $1<\alpha<2-\beta$ (this system may not be competitive). There is no interior steady state and the balance manifold is given in parametric 
form by:

$$
\begin{aligned}
& x_{1}={ }_{2} F_{1}\left[\frac{\alpha}{\alpha-1}, 1, \frac{\beta-2}{\beta-1}, \frac{T}{T^{*}}\right], \\
& x_{2}=T_{2} F_{1}\left[\frac{\alpha}{\alpha-1}, 1, \frac{\beta-2}{\beta-1}, \frac{T}{T^{*}}\right],
\end{aligned}
$$

where $T^{*}=(\beta-1) /(\alpha-1)<0$. Here ${ }_{2} F_{1}$ is the Gaussian hypergeometric function [22, 23, 24] which is defined for $a, b, c, z \in \mathbb{C}$ with the integral form [22]:

$$
{ }_{2} F_{1}(a, b ; c ; z)=\frac{\Gamma(c)}{\Gamma(b) \Gamma(c-b)} \int_{0}^{1} t^{b-1}(1-t)^{c-b-1}(1-t z)^{-a} d t
$$

and $\Gamma$ is the Gamma function. An example of this system is shown in Figure 3a.

In other cases, where the interior steady state exists, the solution will be defined piecewise and joined at the interior steady state (see Figure 3b). The solution in this case, along with the other cases, can be found in [13].

\subsection{Higher order polynomial per-capita growth rates}

We can also consider higher order polynomial functions for the per-capita growth rates $f$ and $g$, as long as our assumptions are still satisfied. This enables the nullclines $f=0$ and $g=0$ to be slightly more complex, affecting the shape of the orbits in the phase plane. In Figure 4, we have the system:

$$
\begin{aligned}
\frac{d x_{1}}{d t} & =x_{1} f\left(x_{1}, x_{2}\right) \\
& =10 x_{1}\left[x_{1}^{2}-\left(x_{2}-1\right)\left(2 x_{2}-6\right)\left(2 x_{2}-1\right)\right] \\
\frac{d x_{2}}{d t} & =x_{2} g\left(x_{1}, x_{2}\right) \\
& =x_{2}\left[14-0.2 x_{1}^{2}-3 x_{2}^{2}\right] .
\end{aligned}
$$

This system satisfies our assumptions in the case where there is a unique interior steady state apart from the final condition (requiring intraspecific competition). In this model, $\frac{\partial f}{\partial x_{1}}>0$ in $\mathbb{R}_{>0}^{2}$. From Figure 4 , we can see that there are no periodic orbits and $x^{*}$ is a stable node. This demonstrates that our requirement of intraspecific competition (A4) is sufficient but not necessary for the existence of a balance manifold. 


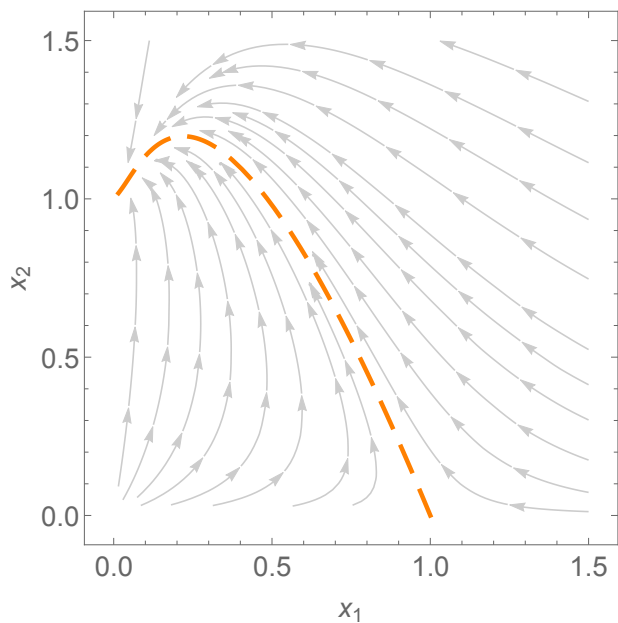

(a) No interior steady state. $\alpha=$ $1.2, \quad \beta=-0.9$.

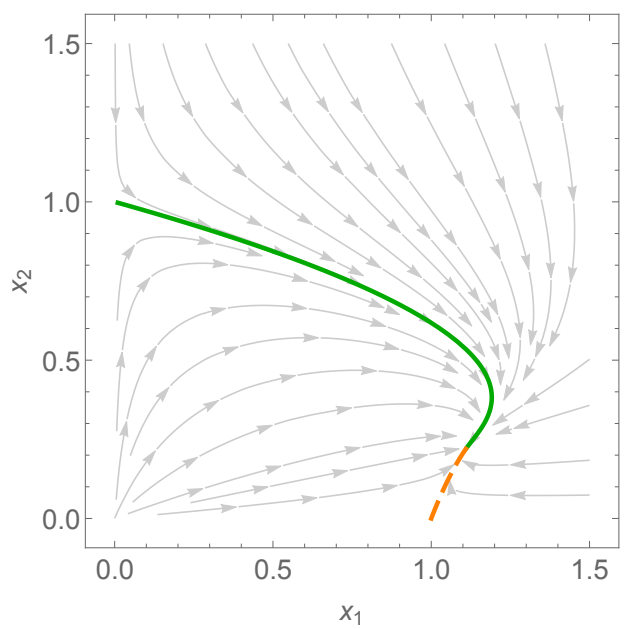

(b) A unique interior steady state. $\alpha=$ $-0.5, \quad \beta=0.7$.

Figure 3: A class of Lotka-Volterra systems [COLOUR: ONLINE ONLY].

Phase planes of the system $\sqrt{12}$, where the solid (green) and dashed (orange) solution curves are the balance manifold. When the interior steady state exists, the solution is defined by joining two orbits together.

\subsection{Facultative mutualism}

In co-operative Lotka-Volterra models, if the interspecific interaction rates are too large, population densities can become unbounded which goes against our assumption that infinity is repelling. Wolin [25] introduced facultative mutualism models for which the orbits are always bounded. A facultative mutualist is a species which can exist without the presence of its mutualistic partner species. We consider a model where both species have a per-capita birth rate which is increased by high recipient densities through a hyperbolic functional response:

$$
\begin{aligned}
& \frac{d x_{1}}{d t}=x_{1}\left(r_{1}-\frac{b_{1} x_{1}}{1+\alpha_{12} x_{2}}-d_{1} x_{1}\right), \\
& \frac{d x_{2}}{d t}=x_{1}\left(r_{2}-\frac{b_{2} x_{2}}{1+\alpha_{21} x_{1}}-d_{2} x_{2}\right),
\end{aligned}
$$

(here all parameters are positive). There is a unique interior steady state $x^{*}$ which always exists and is stable. This model satisfies our assumptions for 

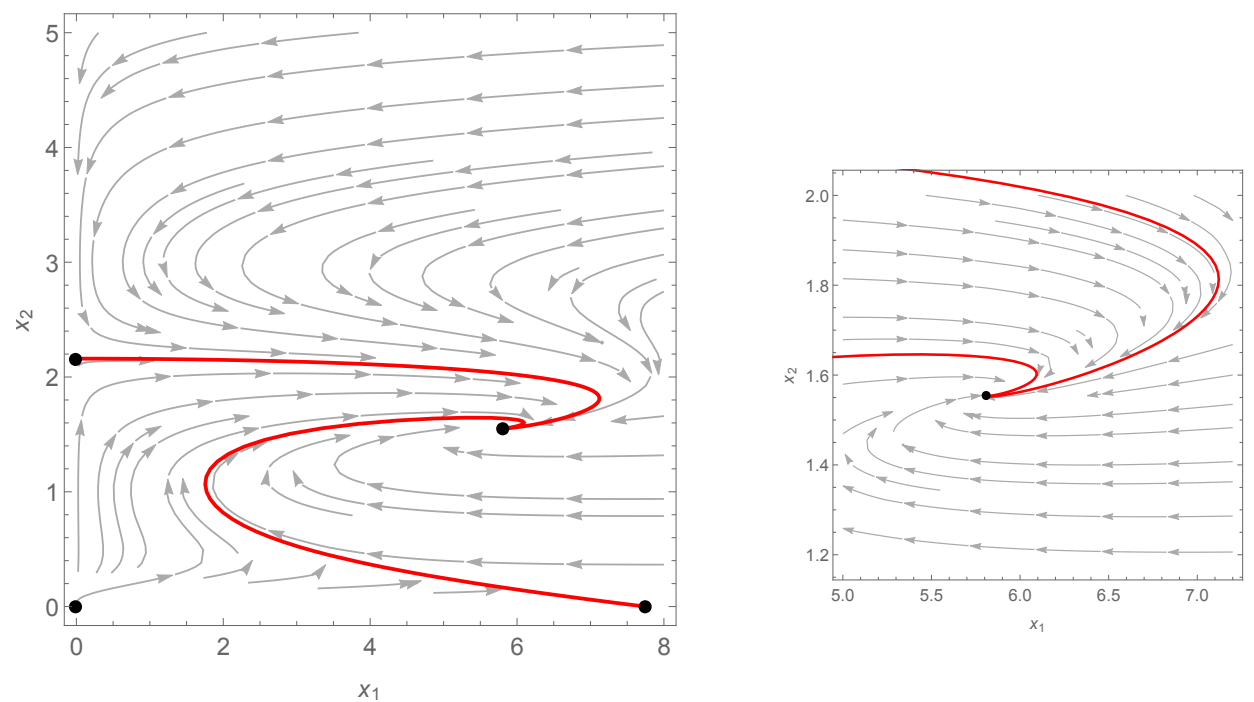

Figure 4: A system with higher order polynomial per-capita growth rates [COLOUR: ONLINE ONLY].

A phase plane of the system (15) with a close-up of the interior steady state in the right figure. The balance manifold is the solid (red) curve and the (black) points are steady states. In this system, there is a unique interior steady state and species 1 does not experience intraspecific competition in $\mathbb{R}_{>0}^{2}$.

the case with one interior steady state. An example of this system, along with its balance manifold, is shown in Figure 5 .

\subsection{Holling type-II Predator-Prey interaction}

Consider the system

$$
\begin{aligned}
& \frac{d x_{1}}{d t}=x_{1}\left[\rho\left(1-\frac{x_{1}}{K}\right)-\frac{\gamma x_{2}}{A+x_{1}}\right], \\
& \frac{d x_{2}}{d t}=x_{2}\left[\frac{\sigma x_{1}}{A+x_{1}}+\mu-\alpha x_{2}\right] .
\end{aligned}
$$

Here, $x_{1}$ is the prey and $x_{2}$ is the predator density, and all parameters are positive. The equation for the prey shows a type-II Holling functional response [26], where there is a maximal feeding rate $\gamma$ for the predator. The model has been modified from the classic predator-prey model as the predator has an alternative food source that supports logistic growth to carrying capacity $\mu / \alpha$ in the absence of prey. An example of this is if the predator is omnivorous [27]. 


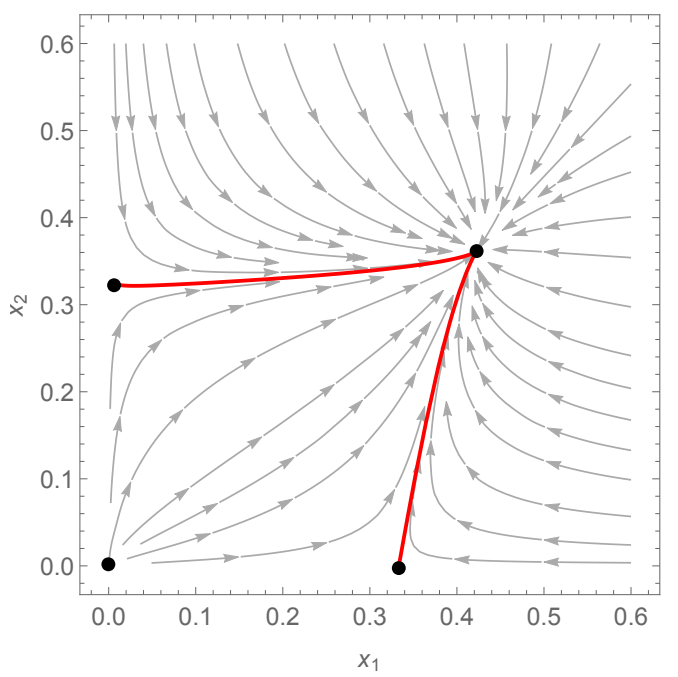

Figure 5: A system with facultative mutualism [COLOUR: ONLINE ONLY]. A phase plane of the co-operative system (16). The balance manifold is the solid (red) curve and the (black) points are steady states.

There is a unique axial steady state on each axis; $(K, 0)$ and $(0, \mu / \alpha)$. There is also intraspecific competition for both species and at most one interior steady state $x^{*}$. In this model, infinity is repelling. Note that $\frac{d x_{1}}{d t}<0$ for any $x_{1}>K$ and $x_{2} \geq 0$. This means there exists some time $T \geq 0$ such that $x_{1}(t) \in[0, K]$ for all $t>T$. In this case, note that $\frac{d x_{2}}{d t} \leq x_{2}\left(\sigma K(A+K)^{-1}+\mu-\alpha x_{2}\right)$ for all $t>T$. The expression on the right hand side is negative when $x_{2}>$ $\alpha^{-1}\left(\sigma K(A+K)^{-1}+\mu\right)$. This means that for any initial condition, there is some time $T_{B}$ such that for all $t>T_{B}$ the solution $\left(x_{1}(t), x_{2}(t)\right)$ lies in the compact box $B=[0, K] \times\left[0, \alpha^{-1}\left(\sigma K(A+K)^{-1}+\mu\right)\right]$. Thus infinity is indeed repelling in this model.

When the parameters of (17) are all positive, the model satisfies our conditions of the existence of a balance manifold in both of the cases where $x^{*}$ does and does not exist. An example of the former case, with the balance manifold depiected, is shown in Figure 6.

\section{Conclusions}

We have provided several computable conditions in Theorems 4.2 and 5.3 which lead to the existence of what we have called the balance manifold in planar Kolmogorov systems, where solutions that are growing from the ori- 

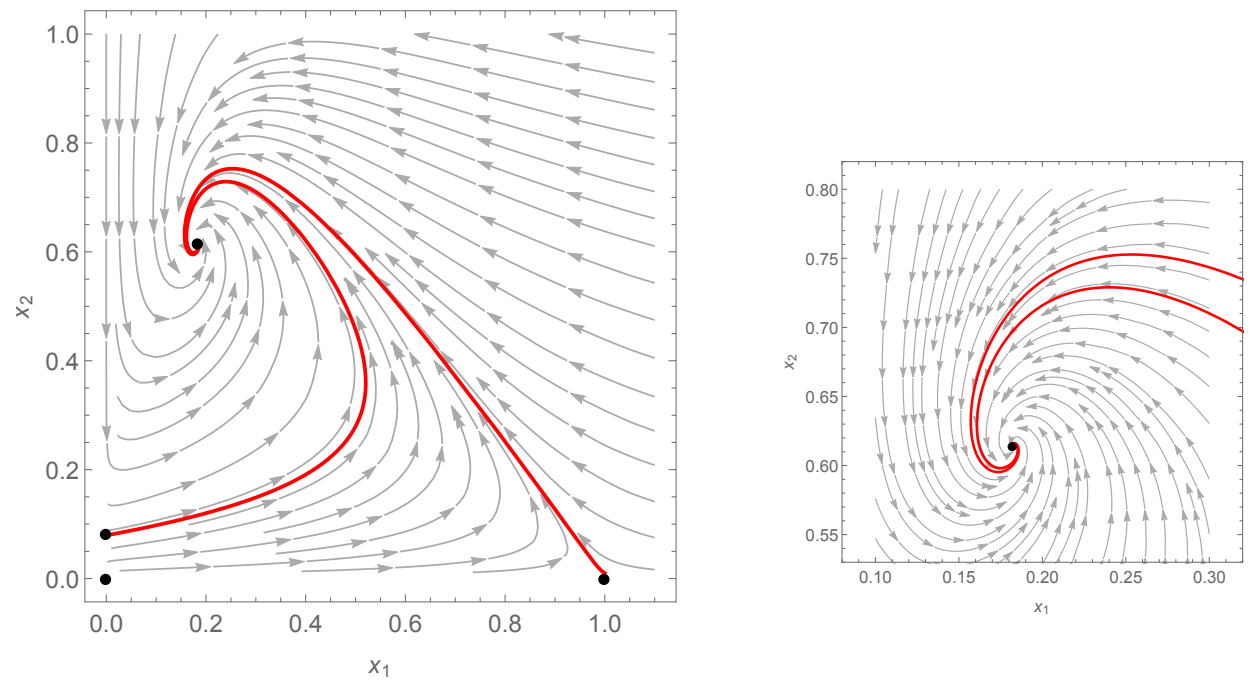

Figure 6: A predator-prey system with a Holling type-II response [COLOUR: ONLINE ONLY].

A phase plane of the system (17) with a close-up of the interior steady state in the right figure. The balance simplex is the solid (red) curve and the (black) points are steady states. In this model of predator-prey dynamics, there is no possibility of periodic orbits since both species always experience intraspecific competition.

gin, with those declining from infinity are balanced. We have discussed the cases where there is at most one interior steady state $x^{*}$. The next logical step would be to consider systems where there is more than one interior steady state. For example, Zhang et al. [28] considered a two-species model with transitions between types of population interactions; the interspecific interactions can change sign with species density. In their model they found up to three interior steady states, an example of which is shown in Figure 7. Unlike our previous examples, in this case the boundary of the basin of repulsion of the origin and of infinity are not equal, but $\mathcal{R}(O) \subset \mathcal{R}(\infty)$ and the balance manifold is $\partial \mathcal{R}(\infty)$ This is why we use the union of these boundaries in Definition 3.7 instead of being equal to both of these boundaries.

Some of the conditions we have provided are necessary for a balance manifold to exist. For example, the requirement that there is exactly one axial steady state on each axis. This condition is equivalent to requiring the existence of the balance manifold in all the 1-dimensional cases of the system. Some of our conditions are sufficient but not necessary for the existence of a balance manifold, such as intraspecific competition in $\mathbb{R}_{>0}^{2}$ (see Figure 6). 


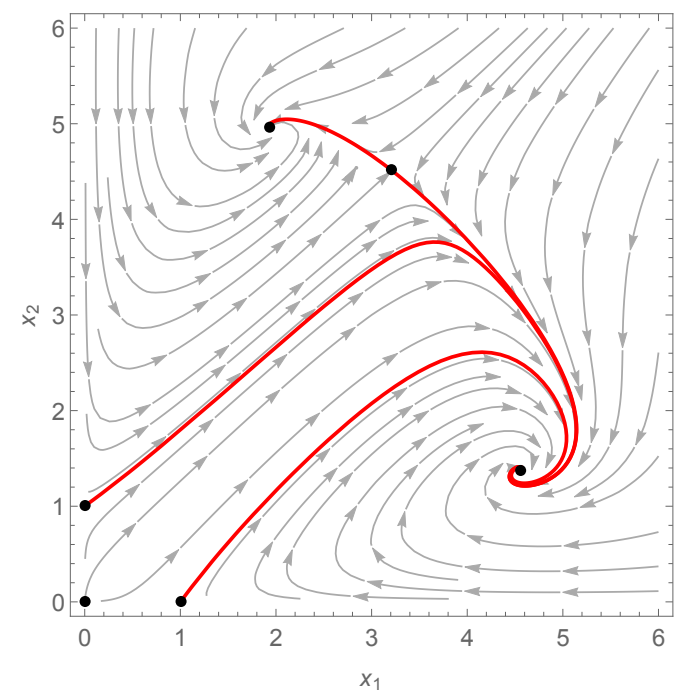

Figure 7: A system with varying interspecific interactions [COLOUR: ONLINE ONLY].

A phase plane of a system from [28, with varying interspecific interactions. The balance simplex is the solid (red) curve and the (black) points are steady states. In this case the boundary of the basin of repulsion of the origin and of infinity are not equal and the balance manifold is $\partial \mathcal{R}(\infty)$.

The balance manifold differs from the competitive carrying simplex as some properties of the carrying simplex no longer hold. For example, in noncompetitive cases where there is an interior steady state, the balance manifold may no longer be smooth at this point. The balance manifold can also have a curvature which changes sign, and may not project 1-to-1 on the line joining both axial steady states. An example of these can be found in Figure 66 where we consider a predator-prey type model.

Finally, the planar balance manifold is an important part of understanding similar manifolds in higher-dimensional Kolmogorov models. For example, where there are 3 species, in seeking to define a balance manifold we need a balance manifold to exist when one of the species is absent, i.e. in a planar model of the kind studied here.

\section{Acknowledgement}

Atheeta Ching was supported by the Engineering and Physical Sciences Research Council, grant number [EP/M507970/1]. 


\section{References}

[1] A. Arneodo, P. Coullet, C. Tresser, Occurence of strange attractors in three-dimensional Volterra equations, Physics Letters A 79 (4) (1980) 259-263.

[2] M. E. Gilpin, Spiral chaos in a predator-prey model, The American Naturalist 113 (2) (1979) 306-308.

[3] J. Vano, J. Wildenberg, M. Anderson, J. Noel, J. Sprott, Chaos in lowdimensional Lotka-Volterra models of competition, Nonlinearity 19 (10) (2006) 2391-2404.

[4] M. W. Hirsch, Systems of differential equations which are competitive or cooperative: III. Competing species, Nonlinearity 1 (1) (1988) 51-71.

[5] M. W. Hirsch, On existence and uniqueness of the carrying simplex for competitive dynamical systems, Journal of Biological Dynamics 2 (2) (2008) 169-179.

[6] O. Diekmann, Y. Wang, P. Yan, Carrying simplices in discrete competitive systems and age-structured semelparous populations, Discrete and Continuous Dynamical Systems 20 (1) (2008) 37-52.

[7] J. Jiang, J. Mierczyński, Y. Wang, Smoothness of the carrying simplex for discrete-time competitive dynamical systems: a characterization of neat embedding, Journal of Differential Equations 246 (4) (2009) 16231672 .

[8] Y. Wang, J. Jiang, Uniqueness and attractivity of the carrying simplex for discrete-time competitive dynamical systems, Journal of Differential Equations 186 (2) (2002) 611-632.

[9] X. Liang, J. Jiang, The dynamical behaviour of type-K competitive Kolmogorov systems and its application to three-dimensional type-K competitive Lotka-Volterra systems, Nonlinearity 16 (3) (2003) 785801.

[10] S. Baigent, Convexity-preserving flows of totally competitive planar Lotka-Volterra equations and the geometry of the carrying simplex, Proceedings of the Edinburgh Mathematical Society 55 (1) (2012) 5363. 
[11] S. Baigent, Geometry of carrying simplices of 3-species competitive Lotka-Volterra systems, Nonlinearity 26 (4) (2013) 1001-1029.

[12] E. C. Zeeman, M. L. Zeeman, An n-dimensional competitive LotkaVolterra system is generically determined by the edges of its carrying simplex, Nonlinearity 15 (6) (2002) 2019-2032.

[13] A. Ching, S. Baigent, The balance simplex in non-competitive 2-species scaled Lotka-Volterra systems, Journal of Biological Dynamics 13 (1) (2019) 128-147.

[14] J. Mierczyński, Smoothness of unordered curves in two-dimensional strongly competitive systems, Applicationes Mathematicae 25 (4) (1999) 449-455.

[15] A. Tineo, On the convexity of the carrying simplex of planar LotkaVolterra competitive systems, Applied Mathematics and Computation 123 (1) (2001) 93-108.

[16] L. Perko, Differential equations and dynamical systems, Vol. 7, Springer Science \& Business Media, 2013.

[17] P. Hartman, Ordinary Differential Equations, Classics in Applied Mathematics, Society for Industrial and Applied Mathematics, 2002.

[18] L. Shilnikov, A. Shilnikov, D. Turaev, L. Chua, Methods of Qualitative Theory in Nonlinear Dynamics. Part I ., World Scientific, 1998.

[19] S. Wiggins, Introduction to applied nonlinear dynamical systems and chaos, Vol. 2, Springer Science \& Business Media, 2003.

[20] L. Shilnikov, A. Shilnikov, D. Turaev, L. Chua, Methods of Qualitative Theory in Nonlinear Dynamics. Part II., World Scientific, 2001.

[21] Y. A. Kuznetsov, Elements of applied bifurcation theory, Vol. 112, Springer Science \& Business Media, 2013.

[22] W. Bailey, Generalized Hypergeometric Series, Cambridge tracts in mathematics and mathematical physics, Stechert-Hafner service agency, 1964. 
[23] H. Bateman, Higher Transcendental Functions [Volumes I-III], McGrawHill Book Company, 1953.

[24] N. J. Vilenkin, A. U. Klimyk, Representation of Lie Groups and Special Functions: Volume 1., Mathematics and its Applications, Kluwer Academic Publishers, 1991.

[25] C. L. Wolin, L. R. Lawlor, Models of facultative mutualism: density effects, The American Naturalist 124 (6) (1984) 843-862.

[26] C. S. Holling, Some characteristics of simple types of predation and parasitism, The Canadian Entomologist 91 (7) (1959) 385-398.

[27] P. Kratina, R. M. LeCraw, T. Ingram, B. R. Anholt, Stability and persistence of food webs with omnivory: is there a general pattern?, Ecosphere 3 (6) (2012) 1-18.

[28] B. Zhang, Z. Zhang, Z. Li, Y. Tao, Stability analysis of a two-species model with transitions between population interactions, Journal of Theoretical Biology 248 (1) (2007) 145-153. 